\title{
Artemether suppresses cell proliferation and induces apoptosis in diffuse large $B$ cell lymphoma cells
}

\author{
XINYING ZHAO $^{1 *}$, XUDONG GUO $^{2,3^{*}}$, WENQIN YUE $^{1}$, JIANMIN WANG $^{1}$, JIANMIN YANG $^{1}$ and JIE CHEN $^{1}$ \\ ${ }^{1}$ Department of Hematology, Changhai Hospital, Second Military Medical University, Shanghai 200168; \\ ${ }^{2}$ Clinical and Translational Research Centre of Shanghai First Maternity \& Infant Health Hospital, Shanghai \\ Key Laboratory of Signaling and Disease Research, Collaborative Innovation Centre for Brain Science, \\ School of Life Science and Technology, Tongji University, Shanghai 200092; ${ }^{3}$ Institute of Regenerative \\ Medicine, East Hospital, Tongji University School of Medicine, Shanghai 200120, P.R. China
}

Received January 4, 2017; Accepted August 23, 2017

DOI: $10.3892 /$ etm.2017.5063

\begin{abstract}
Artemether (ART), a derivative of the well-known anti-malaria drug artemisinin, demonstrates potent anti-cancer activity in various cancer cells, however its effects on lymphoma remain unknown. The present study demonstrated that ART significantly inhibited proliferation of diffuse large B cell lymphoma (DLBCL) in vivo and in vitro, and led to G0/G1 phase arrest. Mechanistic studies demonstrated that ART suppressed the expression of the cell cycle proteins cyclin dependent kinase (CDK) 2, 4, and Cyclin D1, and specifically repressed the proto-oncogene $\mathrm{c}-\mathrm{Myc}$, rather than regulating the extracellular signal-regulated kinase or protein kinase B signaling pathways (two key pathways involved in regulating cell proliferation). In addition, high-concentration ART treatment significantly induced the apoptosis of DLBCL cells by promoting the cleavage of Caspase- 3 and Poly (ADP-ribose) polymerase (PARP) 1. Overall, the data indicated that ART exhibited anti-cancer activity by inhibiting the expression of cell cycle genes and c-Myc, and promoting Caspase- 3 and PARP1 cleavage, which suggested that ART may serve as a dual pharmaceutical for the treatment DLBCL.
\end{abstract}

Correspondence to: Professor Jianmin Yang or Professor Jie Chen, Department of Hematology, Changhai Hospital, Second Military Medical University, 168 Changhai Road, Shanghai 200168, P.R. China

E-mail: chyangjianmin@163.com

E-mail: chenjiedoctor@163.com

*Contributed equally

Abbreviations: ART, artemether; DLBCL, diffuse large B cell lymphoma; NHL, non-Hodgkin lymphoma; VEGF, vascular endothelial growth factor

Key words: artemether, DLBCL, cell cycle arrest, apoptosis, anti-tumor efficacy

\section{Introduction}

Diffuse large B cell lymphoma (DLBCL), which accounts for 30 to $40 \%$ of non-Hodgkin lymphoma (NHL) cases, is the most common malignant lymphoma. Response rates to RCHOP treatment (rituximab, cyclophosphamide, doxorubicin, vincristine, and prednisone) range from 80 to $90 \%$ in patients with low-risk disease (1). However, the response rates for refractory or relapsed patients range from 30 to $60 \%$ with frequent relapses, and salvage chemotherapy is often inadequate for these patients. Thus, there is an urgent need to develop new anti-tumor drugs with better efficacy and lower toxicity that can enhance the chemotherapeutic sensitivity of refractory and relapsed patients.

Botanical drugs are pharmaceuticals of plant origin that generally have multiple targets and fewer side effects than those of traditional medicines. Artemisinin and its derivatives, including artesunate, dihydroartemisnin and artemether (ART), are well-known anti-malaria botanical drugs, and these sesquiterpene lactone compounds contain specific endoperoxide bridges. Abundant experimental and clinical studies have shown that artemisinin and its derivatives are effective in treating malaria with little drug resistance (2). In recent studies, these artemisinin drugs have not only exhibited significant cytotoxicity towards and inhibitory effects on different cancer cells under experimental conditions (3-6), but also increased the recurrence-free survival with well-toleration in colorectal cancer patients and contributed to regression in prostate carcinoma patients $(7,8)$. These findings suggest that artemisinin derivatives may also be promising drugs for treating lymphoma patients. However, the effects of these artemisinin drugs on lymphoma are still unclear.

Intracellular free iron is reported to be more abundant in cancer cells than normal cells (9). Artemisinin and its derivatives can react with intracellular free iron to form cytotoxic free radicals and increase the activity of antioxidant enzymes, promoting apoptosis in cancer cells (10). Furthermore, the expression of genes involved in iron metabolism is positively correlated with the sensitivity of cancer cells to artemisinin treatment (11). Additionally, tumor cells can secrete vascular endothelial growth factor (VEGF) receptors to increase 

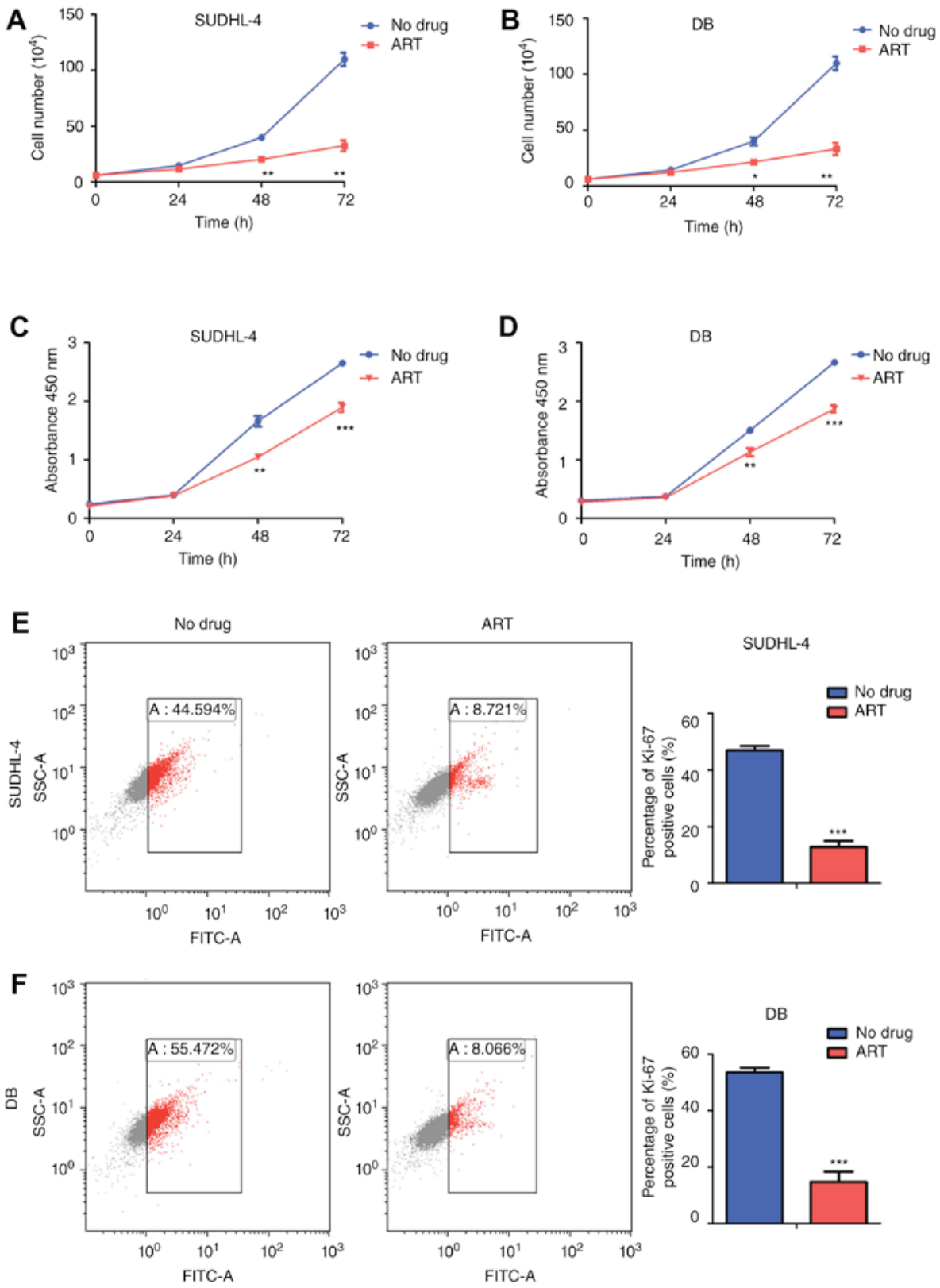

Figure 1. ART inhibits the growth and proliferation of SUDHL-4 and DB cells. (A and B) Cell growth curves of SUDHL-4 (A) and DB cells (B) were generated by cell counting after ART treatment for different durations (24, 48 and $72 \mathrm{~h}$ ). (C and D) CCK8 assays were performed to compare the cell proliferation of SUDHL-4 (C) and DB cells (D) after ART treatment for different durations (24, 48 and $72 \mathrm{~h}$ ). (E and F) Flow cytometry was employed to detect the expression of Ki-67 in SUDHL-4 (E) and DB (F) cells with or without ART treatment for $48 \mathrm{~h}$. Data are presented as the mean \pm standard deviation (n=3). Significance for differences between groups was determined by two-tailed Student's t-test. "*' indicates ART vs. No drug, which was determined by two-tailed Student's t-test. ${ }^{*} \mathrm{P}<0.05 .{ }^{* *} \mathrm{P}<0.01 .{ }^{* * * *} \mathrm{P}<0.001$ vs. no drug. Error bar, standard deviation.

capillary permeability, promote proliferation and migration of endothelial cells and contribute to tumor angiogenesis. Capillary permeability and tumor angiogenesis are reduced by inhibiting VEGF receptors (12). Artemisinin is also shown to inhibit tumor angiogenesis by suppressing the expression of VEGF in treatment of brain glioma (5). Moreover, artemisinin inhibits the proliferation of tumor cells by blocking the apoptosis pathway of P53-independent tumors (13). All these studies indicate that artemisinin and its derivatives are potential anti-tumor drugs, but the detailed mechanisms require further elucidation.

Here, we used two human DLBCL cell lines, SUDHL-4 and DB, to explore the anti-cancer effects of ART (a derivative of artemisinin) on DLBCL cells. Our results showed that ART significantly inhibited the proliferation of DLBCL cells by suppressing the expression of cell cycle-related genes (CDK2, CDK4, and Cyclin D1) and c-Myc, and induced DLBCL cells apoptosis by activating the Caspase-3/PARP1 axis.

\section{Materials and methods}

Cell lines and cell culture. The DLBCL cell lines SUDHL-4 and DB were generously provided by Shanghai Ruijin Hospital (Shanghai, China). Cells were cultured in DMEM (Gibco, USA) supplemented with $10 \%$ fetal bovine serum (Gibco, USA) at $37^{\circ} \mathrm{C}$ in a humidified incubator containing 

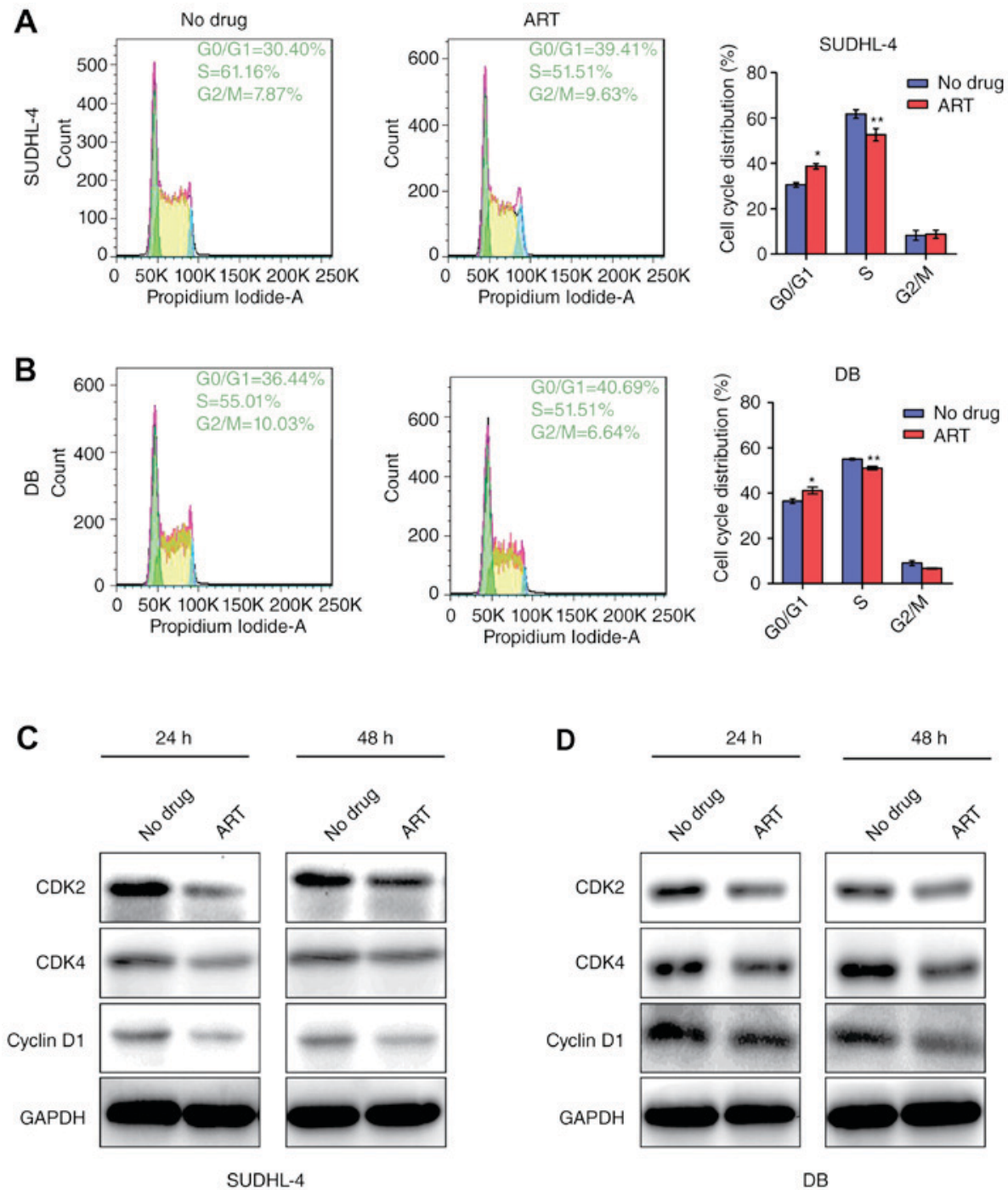

DB

Figure 2. ART treatment results in G0/G1 phase arrest of DLBCL cells and down-regulates cyclin expression. (A and B) Cell cycle distributions SUDHL-4 (A) and DB cells (B) with or without ART treatment were analyzed by flow cytometry. (C and D) Western blotting analysis was used to measure the expression of CDK2, CDK4, and Cyclin D1 in SUDHL-4 (C) and DB (D) cells with or without ART treatment at 24 and $48 \mathrm{~h}$. GAPDH was used as a loading control. Data are presented as the mean \pm standard deviation $(n=3)$. "*, indicates ART vs. No drug, which was determined by two-tailed Student's t-test. "P<0.05. ${ }^{* *} \mathrm{P}<0.01$ vs. no drug. Error bar, standard deviation.

$5 \% \mathrm{CO}_{2}$, supplied with fresh medium every 3 days and subcultured when confluence was reached.

Cell counting assays and Cell Counting Kit-8 (CCK8) analysis. SUDHL-4 and DB cells were seeded into 12-well plates at a density of $6 \times 10^{4}$ cells per well. ART, purchased from Sigma-Aldrich Co. and dissolved in ethanol (Sinopharm Chemical Reagent Co., Ltd.), was added to the medium to reach a final concentration of $0.1 \mathrm{mM}$. Cells with an equal volume of ethanol were used as negative controls. Cell counting assays were performed after 24,48 , and $72 \mathrm{~h}$ of ART treatment. Cell proliferation was assessed in 96-well plates at a density of $3 \times 10^{3}$ cells per well using the CCK8 assay (DOJINDO, Japan).

Ki-67 detection. DLBCL cells were treated with ART $(0.1 \mathrm{mM})$ and collected after $48 \mathrm{~h}$. Cells were fixed by $2 \%$ PFA for $30 \mathrm{~min}$. After permeabilization, the cells were further stained with $\mathrm{Ki}-67$ (Abcam, ab66155) at $37^{\circ} \mathrm{C}$ for $60 \mathrm{~min}$ and then stained with FITC-labeled Goat Anti-Rabbit IgG (H+L)
(Beyotime Biotechnology) at $37^{\circ} \mathrm{C}$ for $60 \mathrm{~min}$ in the dark before flow cytometric analysis.

Cell cycle analysis. SUDHL-4 and DB cells were seeded into 12-well plates at a density of $6 \times 10^{4}$ cells per well and treated with ART $(0.1 \mathrm{mM})$ for $48 \mathrm{~h}$. Cells were washed twice with phosphate buffered saline (PBS) and then resuspended with precooled $70 \%$ ethanol overnight at $4^{\circ} \mathrm{C}$. After centrifugation, the pellets were washed twice with precooled PBS. Each sample was mixed with $500 \mu$ of staining buffer, $25 \mu \mathrm{l}$ propidium iodide (PI) staining solution and $10 \mu 1$ RNase A (Beyotime Biotechnology) and incubated for $30 \mathrm{~min}$ in the dark at $37^{\circ} \mathrm{C}$. The cell cycle distribution was evaluated by flow cytometry.

Cell apoptosis assay. SUDHL-4 and DB cells were seeded into 12-well plates at a density of $6 \times 10^{4}$ cells per well and treated with ART $(0.3 \mathrm{mM})$ for $48 \mathrm{~h}$. Cells with an equal volume of ethanol were used as negative controls. All cells were collected 
after a $48 \mathrm{~h}$ treatment and then washed twice with PBS. After centrifugation, each sample was mixed with $195 \mu 1$ staining buffer, $5 \mu \mathrm{l}$ PI staining solution and $5 \mu \mathrm{l}$ Annexin V-FITC solution (KeyGEN Biotech.) and incubated for $20 \mathrm{~min}$ in the dark at $37^{\circ} \mathrm{C}$ before flow cytometry analysis.

Western blotting. RIPA buffer (KeyGEN Biotech.) was used to lyse the cells, and protein concentration was quantified by the BCA method. An equal amount of the extracted protein was separated by $10 \%$ SDS-PAGE and transferred onto PVDF membranes. The membranes were blocked with 3\% BSA for $1 \mathrm{~h}$ at room temperature and then incubated with the primary antibodies overnight at $4^{\circ} \mathrm{C}$. After three washes with TBST, the membranes were incubated with secondary antibodies $(1: 3,000)$ for $1 \mathrm{~h}$ at room temperature. The membranes were washed with TBST three times before visualization by a chemiluminescence system. Antibodies used in this work: Mouse anti-CDK2 (SC-6248, Santa Cruz Biotechnology), rabbit anti-CDK4 (SC-260, Santa Cruz Biotechnology), rabbit anti-Cyclin D1 (SC-718, Santa Cruz Biotechnology), mouse anti-c-Myc (ab32, Abcam), rabbit anti-ERK (4695S, Cell Signaling Technology), rabbit anti-P-ERK (4370S, Cell Signaling Technology), rabbit anti-AKT (4685S, Cell Signaling Technology), rabbit anti-P-AKT (4060L, Cell Signaling Technology), rabbit anti-GAPDH (47724, Santa Cruz Biotechnology), rabbit anti-cleaved-Caspase-3 (9661S, Cell Signaling Technology), rabbit anti-Caspase-3 (9662S, Cell Signaling Technology), rabbit anti-cleaved-PARP1 (CY5035, Abways Technology), mouse anti-PARP1 (SC-74469X, Santa Cruz Biotechnology), HRP-Ms (7076, Cell Signaling Technology), and HRP-Rb (7074, Cell Signaling Technology).

Tumor xenografts. Six-week-old NOD-SCID mice were purchased from the National Resource Centre for Rodent Laboratory Animals of China. Initially, $1 \times 10^{7} \mathrm{DB}$ cells suspended in $100 \mu \mathrm{l}$ with 1 part matrigel and 2 part DMEM were injected subcutaneously into the left and right thighs of the mice. On day 16 after tumor injection, the mice were injected intraperitoneally with ART (200 mg/kg) every day until day 25 . Then, the mice were sacrificed at day 25 post-injection.

Statistical analysis. Statistical analysis was performed using SPSS 11.0 software, and the results were presented as the mean \pm standard deviation from triplicate experiments. Significance differences were determined by two-tailed Student's t-test, and $\mathrm{P}<0.05$ was considered statistically significant.

\section{Results}

ART treatment inhibits the growth and proliferation of SUDHL-4 and DB cells. Preliminary experiments indicated that $0.1 \mathrm{mM}$ ART treatment for $48 \mathrm{~h}$ led to half maximal inhibition of SUDHL-4 and DB cells. There was no significant abnormality in the growth of SUDHL-4 and DB cells after ART $(0.1 \mathrm{mM})$ treatment for $24 \mathrm{~h}$. Following ART $(0.1 \mathrm{mM})$ treatment for $48 \mathrm{~h}$, the number of SUDHL-4 cells was $(20.26 \pm 2.58) \times 10^{4}$ and the number of DB cells was $(21.44 \pm 2.70) \times 10^{4}$, which were much lower compared with those of the negative controls, $(39.99 \pm 2.38) \times 10^{4}$ for SUDHL-4 cells and $(40.11 \pm 6.36) \times 10^{4}$
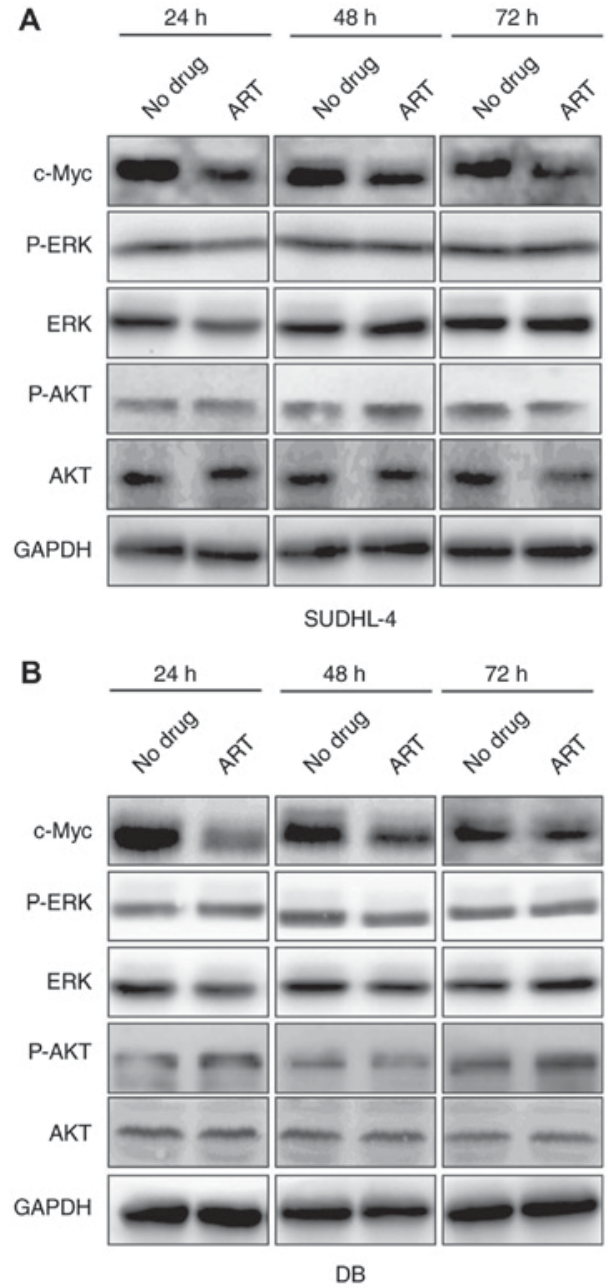

Figure 3. ART specifically inhibits the expression of c-Myc. (A and B) Western blotting analysis was used to measure the expression of c-Myc, P-ERK/ERK, and P-AKT/AKT in SUDHL-4 (A) and DB (B) cells with or without ART treatment at 24,48 , and $72 \mathrm{~h}$ from triplicate experiments. GAPDH was used as a loading control.

for DB cells. These results showed that ART treatment for 48 h significantly inhibited the growth of SUDHL-4 and DB cells. The percentages of growth inhibition in SUDHL-4 and DB cells were $49.35 \pm 5.33 \%$ and $46.37 \pm 1.69 \%$, respectively (Fig. 1A and B). For the $72 \mathrm{~h}$ treatment, the growth inhibition percentages were $70.63 \pm 5.53 \%$ for SUDHL-4 cells and $70.05 \pm 6.22 \%$ for DB cells, showing more significant inhibitory effects on DLBCL cell growth (Fig. 1A and B). CCK8 analysis indicated that ART significantly inhibited the proliferation of DLBCL cells after 48 and $72 \mathrm{~h}$ treatments (Fig. 1C and D). The DLBCL cells exposed to ART treatment had significantly decreased Ki-67 expression, which confirmed the inhibition of DLBCL cell proliferation (Fig. 1E and F). These results indicated that treatment with ART had a significant inhibitory effect on cell growth and proliferation in DLBCL cells.

ART treatment results in $G 0 / G 1$ phase arrest of $D L B C L$ cells and down-regulates cyclin expression. To determine whether the growth delay was due to arrest in any specific cell cycle phase, we used flow cytometry to compare the cell cycle distribution of untreated cells vs. that of cells 

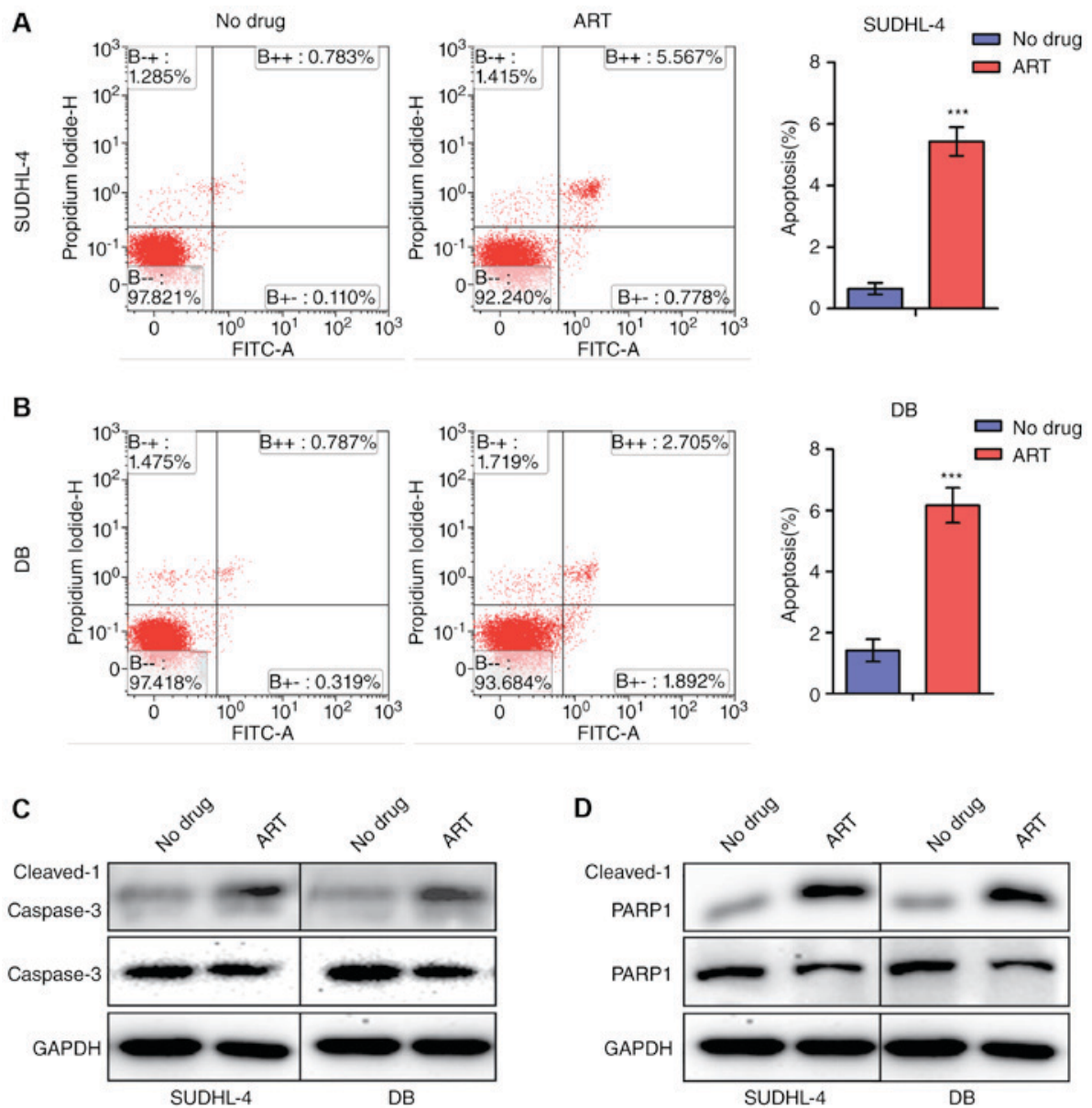

Figure 4. High-concentration ART treatment induces the apoptosis of DLBCL cells by activating the Caspase-3/PARP1 axis. (A and B) Apoptosis of untreated and $0.3 \mathrm{mM}$ ART-treated SUDHL-4 (A) and DB (B) cells was analyzed by flow cytometry. (C and D) Western blotting analysis was used to measure the expression of cleaved-Caspase-3/Caspase (C) and cleaved-PARP1/PARP1 (D) in SUDHL-4 and DB cells with or without ART treatment. Data are presented as the mean \pm standard deviation $(\mathrm{n}=3) .{ }^{{ }^{*},}$ indicates ART vs. No drug, which was determined by two-tailed Student's t-test. ${ }^{* * *} \mathrm{P}<0.001$ vs. no drug. Error bar, standard deviation.

treated with $0.1 \mathrm{mM}$ ART. Our data recorded severe arrest in G0/G1 phase of SUDHL-4 cells $(38.73 \pm 1.25 \%)$ and DB cells $(41.12 \pm 1.56 \%)$ after $0.1 \mathrm{mM}$ ART treatment for $48 \mathrm{~h}$ compared with that of the untreated cells $(30.67 \pm 1.45 \%$ for SUDHL-4 cells and $36.46 \pm 1.05 \%$ for DB cells) (Fig. 2A and B). Additionally, the percentages of S-phase cells were decreased by $9.12 \pm 0.82 \%$ (SUDHL-4) and $4.03 \pm 1.13 \%$ (DB) $(\mathrm{P}<0.05)$ (Fig. 2A and B). Taken together, the results showed that ART treatment arrested cells in G0/G1 phase, leading to failure to enter S-phase.

CDK2, CDK4 and Cyclin D1 play important roles in G1/S transition, which is positively related to cell proliferation. Reduction of these cyclins indicates that cells are arrested in G1 phase and cell proliferation is restrained. To determine how ART affected cell cycle distribution of DLBCL cells, we measured the CDK2, CDK4 and Cyclin D1 levels and found that these proteins were substantially down-regulated with ART treatment for 24 and $48 \mathrm{~h}$ (Fig. 2C and D). These results were consistent with the cell cycle detection by flow cytometry.

ART specifically inhibits the expression of c-Myc. c-Myc is a proto-oncogene that is involved in many malignant behaviors of cancers including proliferation, invasion and activation of cancer signaling pathways (14). ERK and AKT are important kinases in MAPK signaling and PI3K signaling respectively, which are important for tumor progression $(15,16)$. Our results showed that c-Myc expression was dramatically down-regulated after SUDHL-4 and DB cells were treated for different durations (24, 48 and 72 h) (Fig. 3A and B). However, ART treatment had no significant effect on the expression of P-ERK/ERK and P-AKT/AKT at 24, 48 or $72 \mathrm{~h}$ (Fig. 3A and $\mathrm{B}$ ), suggesting that ART-mediated inhibition of cell proliferation was predominantly regulated by decreasing the expression of c-Myc, rather than the other two key signaling pathways involved in cell proliferation.

High-concentration ART treatment induces apoptosis of DLBCL cells by activating the Caspase-3/PARPI axis. To determine whether ART affected DLBCL cell apoptosis, we evaluated the intensity of apoptosis by Annexin V-FITC and PI staining using flow cytometry. Treating SUDHL-4 and DB cells with ART $(0.1 \mathrm{mM})$ did not make any notable difference on the percentages of apoptotic cells, compared with the negative controls (data not shown). While the percentages of apoptotic cells were significantly increased to $5.03 \pm 0.59 \%$ in SUDHL- 4 cells and $6.83 \pm 1.08 \%$ in DB cells after treatment with ART $(0.3 \mathrm{mM})(\mathrm{P}<0.05)$ (Fig. $4 \mathrm{~A}$ and $\mathrm{B})$. Furthermore, our 
results showed that the active forms of Caspase- 3 and PARP1 (cleaved-Caspase-3 and cleaved-PARP1) were significantly increased after ART treatment, suggesting that ART promoted the cleavage of Caspase-3 and PARP1 (Fig. 4C and D). These results indicated that ART induced apoptosis by activating the Caspase-3/PARP1 axis.

ART treatment inhibits DLBCL cell growth in vivo. To further determine the in vivo effects of ART, we constructed transplanted tumor models using six-week-old NOD-SCID mice. Equal amounts of DB cells were injected subcutaneously into left and right thighs of mice. Then, the mice were injected intraperitoneally with ART $(200 \mathrm{mg} / \mathrm{kg})$ once a day from day 16 to day 25 post-injection. Our results showed that the ART-treated groups presented smaller tumor volumes (Fig. 5A and B) and lighter tumor weights (Fig. 5C) than those of the control groups, which suggested that ART alleviated the tumor burden of mice in vivo.

\section{Discussion}

Recent studies have shown that artemisinin drugs exhibited significant cytotoxicity and inhibitory effects on cancer cells, including leukemia, stomach cancer, breast cancer, and pancreatic cancer cells (3-6). The anti-tumor effects and mechanisms of ART on lymphoma remain unexplored. Our results showed that ART significantly inhibited the proliferation of DLBCL cells and arrested these cells in G0/G1 phase. Moreover, increased concentrations of ART induced apoptosis of DLBCL cells. Together, our data first indicated that ART treatment significantly inhibited proliferation, promoted G0/G1 phase arrest and induced apoptosis of DLBCL cells, suggesting that ART is a potential drug to DLBCL treatment.

Artemisinin has been reported to inhibit tumor angiogenesis by suppressing VEGF expression or to treat P53-independent tumors by blocking the apoptosis pathway (13), but the mechanisms of ART-mediated inhibition of lymphoma cell proliferation remain unclear. CDK2 is a crucial cyclin-dependent kinase and essential for G1/S transition. This protein maintains $\mathrm{Rb}$ phosphorylation in late G1 phase to ensure cells enter S phase (17) and is thus considered a potential target for anti-tumor treatments (18). Cyclin D1/CDK4 can act on Cyclin D1-pRb to regulate the G1/S transition $(19,20)$. Our results showed that ART-treated DLBCL cells had decreased expression of three cell cycle-dependent proteins (CDK2, CDK4, and Cyclin D1), indicating that ART treatment arrested DLBCL cells in G1 phase and inhibited proliferation by suppressing the expression of cell cycle proteins. A previous report showed that inhibition of miR-34a abolished the ART-mediated CDK4 down-regulation and cell cycle arrest (21). Moreover, transcription factors including mTOR, NF- $\mathrm{KB}$, and CREB are reported to be involved in the ART-mediated inhibition of proliferation (22-24). Thus, miRNAs and these transcription factors may be key mediators for ART in down-regulating cell cycle-related gene expression in DLBCL.

The proto-oncogene c-Myc plays major roles in the cell proliferation, cell growth regulation, protein synthesis, and cell adhesion of tumor cells (14). Previous reports

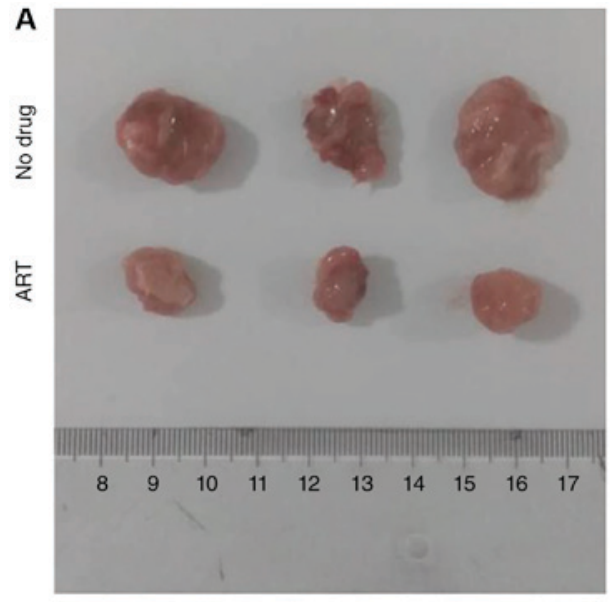

B
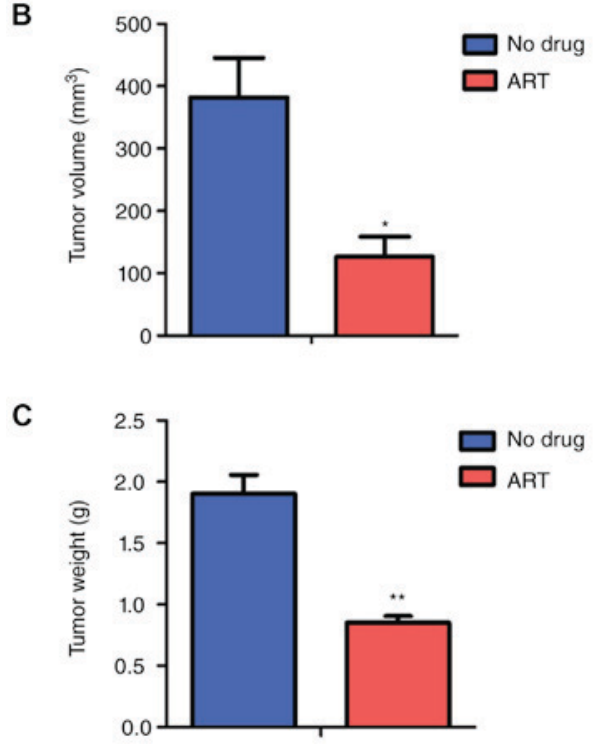

Figure 5. ART treatment inhibits DLBCL cell growth in vivo. (A and B) Representative images (A) and volume statistics (B) of tumors with or without ART treatment in xenografts. (C) Weight statistics of tumors with or without ART treatment in xenografts. Data are presented as the mean \pm standard deviation $(n=3)$. ${ }^{* *}$ indicates ART vs. No drug, determined by two-tailed Student's t-test. ${ }^{*} \mathrm{P}<0.05 .{ }^{* *} \mathrm{P}<0.01$ vs. no drug. Error bar,

have indicated that artemisinin showed potent anti-cancer activity in cells overexpressing c-Myc (25) and induced cell cycle arrest and apoptosis in prostate cancer cells by inhibiting c-Myc (26). ERK signaling, a key signal pathway from surface receptors to the nucleus, is related to progression of various neoplastic diseases (15,27-29). AKT signaling is also involved in the regulation of cell proliferation, differentiation, apoptosis and migration by regulating its downstream target proteins Bad, Caspase9, NF- $\mathrm{BB}$, GSK-3 and others via phosphorylation (16,30-32). We also found that ART treatment significantly decreased the expression level of c-Myc in DLBCL. However, ART treatment did not affect the expression and the phosphorylation of two key kinases, ERK and AKT. These results further confirmed that c-Myc was a key downstream factor of ART in inhibiting DLBCL cell proliferation. Moreover, the data indicated that ART induced DLBCL cell apoptosis by activating the cleavage of Caspase-3 and PARP1. In summary, we elucidated the critical mechanisms underlying proliferation inhibition and 
apoptosis induction by ART in DLBCL cells, indicating ART may be an alternative anti-cancer drug for DLBCL treatment.

Drug resistance and relapse of DLBCL are major challenges to clinical treatment. Hematopoietic stem cell transplant (HSCT) may improve the outcome of patients with relapsed or refractory DLBCL. However, HSCT availability is often limited by patient age, treatment-related morbidities, and poor performance status in many cases (33). Therefore, novel targeted therapies are urgently needed. Natural pharmaceuticals from plants have been increasingly tested due to their multiple targets and few side effects. Recent reports showed that ART could inhibit angiogenesis and reverse chemoresistance $(5,34)$. Our results demonstrated that the natural botanical ART significantly inhibited the proliferation and induced apoptosis of DLBCL cells, suggesting that ART might be a promising combined pharmaceutical with conventional chemotherapeutics to combat chemoresistance and relapse of DLBCL.

\section{Acknowledgements}

This work was supported by the National Natural Science Foundation of China (81570179 and 81170499 to J. C).

\section{References}

1. Feugier P, Van Hoof A, Sebban C, Solal-Celigny P, Bouabdallah R, Fermé C, Christian B, Lepage E, Tilly H, Morschhauser F, et al: Long-term results of the R-CHOP study in the treatment of elderly patients with diffuse large B-cell lymphoma: A study by the Groupe d'Etude des Lymphomes de l'Adulte. J Clin Oncol 23: 4117-4126, 2005.

2. Grandesso F, Nabasumba C, Nyehangane D, Page AL, Bastard M, De Smet M, Boum Y and Etard JF: Performance and time to become negative after treatment of three malaria rapid diagnostic tests in low and high malaria transmission settings. Malar J 15: 496, 2016.

3. Jia G, Kong R, Ma ZB, Han B, Wang YW, Pan SH, Li YH and Sun B: The activation of c-Jun $\mathrm{NH}_{2}$-terminal kinase is required for dihydroartemisinin-induced autophagy in pancreatic cancer cells. J Exp Clin Cancer Res 33: 8, 2014.

4. Shahbazfar AA, Zare P, Ranjbaran M, Tayefi-Nasrabadi H, Fakhri O, Farshi Y, Shadi S and Khoshkerdar A: A survey on anticancer effects of artemisinin, iron, miconazole, and butyric acid on 5637 (bladder cancer) and 4T1 (Breast cancer) cell lines. J Cancer Res Ther 10: 1057-1062, 2014.

5. Wu ZP, Gao CW, Wu YG, Zhu QS, Yan Chen, Xin Liu and Chuen Liu: Inhibitive effect of artemether on tumor growth and angiogenesis in the rat C6 orthotopic brain gliomas model. Integr Cancer Ther 8: 88-92, 2009.

6. Gerhardt T, Jones R, Park J, Lu R, Chan HW, Fang Q, Singh N and Lai $\mathrm{H}$ : Effects of antioxidants and pro-oxidants on cytotoxicity of dihydroartemisinin to Molt-4 human leukemia cells. Anticancer Res 35: 1867-1871, 2015.

7. Krishna S, Ganapathi S, Ster IC, Saeed ME, Cowan M, Finlayson C, Kovacsevics H, Jansen H, Kremsner PG, Efferth T and Kumar D A randomised, double blind, placebo-controlled pilot study of oral artesunate therapy for colorectal cancer. EBioMedicine 2: 82-90, 2015.

8. Michaelsen FW, Saeed ME, Schwarzkopf J and Efferth T: Activity of Artemisia annua and artemisinin derivatives, in prostate carcinoma. Phytomedicine 22: 1223-1231, 2015.

9. Shterman N, Kupfer B and Moroz C: Comparison of transferrin receptors, iron content and isoferritin profile in normal and malignant human breast cell lines. Pathobiology 59: 19-25, 1991.

10. Efferth T, Benakis A, Romero MR, Tomicic M, Rauh R, Steinbach D, Häfer R, Stamminger T, Oesch F, Kaina B and Marschall M: Enhancement of cytotoxicity of artemisinins toward cancer cells by ferrous iron. Free Radic Biol Med 37: 998-1009, 2004.
11. Ooko E, Saeed ME, Kadioglu O, Sarvi S, Colak M, Elmasaoudi K, Janah R, Greten HJ and Efferth T: Artemisinin derivatives induce iron-dependent cell death (ferroptosis) in tumor cells. Phytomedicine 22: 1045-1054, 2015.

12. Carmeliet P and Jain RK: Molecular mechanisms and clinical applications of angiogenesis. Nature 473: 298-307, 2011.

13. Pang Y, Qin G, Wu L, Wang X and Chen T: Artesunate induces ROS-dependent apoptosis via a Bax-mediated intrinsic pathway in Huh-7 and Hep3B cells. Exp Cell Res 347: 251-260, 2016.

14. Stine ZE, Walton ZE, Altman BJ, Hsieh AL and Dang CV: MYC, metabolism, and cancer. Cancer Discov 5: 1024-1039, 2015.

15. Che N, Ma Y and Xin Y: Protective role of fucoidan in cerebral ischemia-reperfusion injury through inhibition of MAPK signaling pathway. Biomol Ther (Seoul) 25: 272-278, 2017.

16. Guo JR, Li W, Wu Y, Wu LQ, Li X, Guo YF, Zheng XH, Lian XL, Huang HF and Chen YZ: Hepatocyte growth factor promotes proliferation, invasion, and metastasis of myeloid leukemia cells through PI3K-AKT and MAPK/ERK signaling pathway. Am J Transl Res 8: 3630-3644, 2016.

17. Roskoski R Jr: Cyclin-dependent protein kinase inhibitors including palbociclib as anticancer drugs. Pharmacol Res 107: 249-275, 2016.

18. Shapiro GI: Cyclin-dependent kinase pathways as targets for cancer treatment. J Clin Oncol 24: 1770-1783, 2006.

19. Park YG, Park S, Lim SO, Lee MS, Ryu CK, Kim I and Cho-Chung Y: Reduction in cyclin D1/Cdk4/retinoblastoma protein signaling by CRE-decoy oligonucleotide. Biochem Biophys Res Comm 281: 1213-1219, 2001.

20. Chohan TA, Qian H, Pan Y and Chen JZ: Cyclin-dependent kinase-2 as a target for cancer therapy: Progress in the development of CDK2 inhibitors as anti-cancer agents. Curr Med Chem 22: 237-263, 2015.

21. Hargraves KG, He L and Firestone GL: Phytochemical regulation of the tumor suppressive microRNA, miR-34a, by p53-dependent and independent responses in human breast cancer cells. Mol Carcinog 55: 486-498, 2016.

22. Odaka Y, Xu B, Luo Y, Shen T, Shang C, Wu Y, Zhou H and Huang S: Dihydroartemisinin inhibits the mammalian target of rapamycin-mediated signaling pathways in tumor cells. Carcinogenesis 35: 192-200, 2014

23. Hu W, Chen SS, Zhang JL, Lou XE and Zhou HJ: Dihydroartemisinin induces autophagy by suppressing NF- $\kappa \mathrm{B}$ activation. Cancer Lett 343: 239-248, 2014.

24. Kim HG, Yang JH, Han EH, Choi JH, Khanal T, Jeong MH, Jeong TC and Jeong HG: Inhibitory effect of dihydroartemisinin against phorbol ester-induced cyclooxygenase-2 expression in macrophages. Food Chem Toxicol 56: 93-99, 2013.

25. Lu JJ, Meng LH, Shankavaram UT, Zhu CH, Tong LJ, Chen G, Lin LP, Weinstein JN and Ding J: Dihydroartemisinin accelerates c-MYC oncoprotein degradation and induces apoptosis in c-MYC-overexpressing tumor cells. Biochem Pharmacol 80: 22-30, 2010.

26. Morrissey C, Gallis B, Solazzi JW, Kim BJ, Gulati R, Vakar-Lopez F, Goodlett DR, Vessella RL and Sasaki T: Effect of artemisinin derivatives on apoptosis and cell cycle in prostate cancer cells. Anticancer Drugs 21: 423-432, 2010.

27. Chung TW, Choi H, Lee JM, Ha SH, Kwak CH, Abekura F, Park JY, Chang YC, Ha KT, Cho SH, et al: Oldenlandia diffusa suppresses metastatic potential through inhibiting matrix metalloproteinase-9 and intercellular adhesion molecule-1 expression via p38 and ERK1/2 MAPK pathways and induces apoptosis in human breast cancer MCF-7 cells. J Ethnopharmacol 195: 309-317, 2017.

28. Youn DH, Park J, Kim HL, Jung Y, Kang JW, Jeong MY, Sethi G, Ahn KS and Um JY: Chrysophanic acid reduces testosterone-induced benign prostatic hyperplasia in rats by suppressing $5 \alpha$-reductase and extracellular signal-regulated kinase. Oncotarget 8: 9500-9512, 2017.

29. Zuo WH, Zeng P, Chen X, Lu YJ, Li A and Wu JB: Promotive effects of bone morphogenetic protein 2 on angiogenesis in hepatocarcinoma via multiple signal pathways. Sci Rep 6: 37499, 2016.

30. Feng X, Jiang J, Shi S, Xie H, Zhou L and Zheng S: Knockdown of miR-25 increases the sensitivity of liver cancer stem cells to TRAIL-induced apoptosis via PTEN/PI3K/Akt/Bad signaling pathway. Int J Oncol 49: 2600-2610, 2016. 
31. Gao Y, Xiao X, Zhang C, Yu W, Guo W, Zhang Z, Li Z, Feng X, Hao J, Zhang K, et al: Melatonin synergizes the chemotherapeutic effect of 5-fluorouracil in colon cancer by suppressing PI3K/AKT and NF- $\mathrm{kB} / \mathrm{iNOS}$ signaling pathways. J Pineal Res 62, 2017 doi: 10.1111/jpi.12380.

32. Wang L, Zhang S, Cheng H, Lv H, Cheng G and Ci X: Nrf2-mediated liver protection by esculentoside A against acetaminophen toxicity through the AMPK/Akt/GSK3 $\beta$ pathway. Free Radic Biol Med 101: 401-412, 2016.
33. Philip T, Guglielmi C, Hagenbeek A, Somers R, Van der Lelie H, Bron D, Sonneveld P, Gisselbrecht C, Cahn JY, Harousseau JL, et al: Autologous bone marrow transplantation as compared with salvage chemotherapy in relapses of chemotherapy-sensitive non-Hodgkin's lymphoma. N Engl J Med 333: 1540-1545, 1995.

34. Reungpatthanaphong P and Mankhetkorn S: Modulation of multidrug resistance by artemisinin, artesunate and dihydroartemisinin in K562/adr and GLC4/adr resistant cell lines. Biol Pharm Bull 25: 1555-1561, 2002. 